\title{
Correction to: Biochemical determinants of litter quality in 15 species of Sphagnum
}

\author{
Fia Bengtsson • Håkan Rydin • Tomáš Hájek
}

Published online: 10 April 2019

(C) Springer Nature Switzerland AG 2019

\section{Correction to: Plant Soil (2018) 425, 161-176 \\ https://doi.org/10.1007/s11104-018-3579-8}

There were mistakes in the data of three variables used in this study. The corrections listed below have minor effects on statistics and $p$-values, but do not change any conclusions. Correct data are stored in the Dryad repository (https://doi.org/10.5061/dryad.4f8d2.2).

\section{Loss in the lab}

Most samples of the variable "labloss $2 b$ " differed $0.6-0.7 \%$ from the correct values. The errors in two samples were larger (MG2 decrease $1.3 \%$, and TE3 increase $4.9 \%$ ).

The online version of the original article can be found at https://doi.org/10.1007/s11104-018-3579-8

F. Bengtsson $(\bowtie) \cdot$ H. Rydin

Department of Plant Ecology and Evolution, Evolutionary

Biology Centre, Uppsala University, Norbyvägen 18D, SE-752

36 Uppsala, Sweden

e-mail: fia.bengtsson@ebc.uu.se

T. Hájek

Department of Experimental Plant Biology, Faculty of Science, University of South Bohemia, Branišovská 1760, CZ-370

05 České Budějovice, Czech Republic

T. Hájek

Department of Functional Ecology, Institute of Botany of the Czech Academy of Sciences, Dukelská 135, CZ-379 82 Třeboň, Czech Republic

\section{Loss in the field}

One sample of S. fuscum, FU13, had a value for "lossfield" that should have been excluded due to ingrowth of roots in the litterbag.

\section{CN ratio}

Some C:N ratio values had been sorted wrongly within species. Because some numbers have moved between the bog and fen samples of S. fuscum, and between the open bog and pine bog samples of $S$. magellanicum, these have changes in averages (S. fus. Bog $-27.1 ; \mathrm{S}$. fus. Fen +27.1; S. mag. Open -1.1 ; S. mag. Pine +1.1).

Specific changes in the text:

Abstract, right column, line 9 "0.57" should read "0.56" p. 167

left column, line 44 " 0.57 " should read " 0.56 ". right column, line 2 " 0.48 " should read " 0.38 ".

line 3 "0.006" should read "0.009", and "59" should read " 57 ".

line 28 "93" should read "96" and "-1.67" should read " -1.74 ".

line 36 " 0.56 " should read " 0.55 ", "0.59" should read " 0.58 ".

line 45 " 0.005 " should read "0.002", "0.5" should read "0.7". line 47 " 0.003 " should read " 0.0004 ", and "0.6" should read " 0.9 ".

line 48- "...carbon concentration, $\mathrm{CN}$ ratio and phosphorus concentration ( $\mathrm{r}=-0.33,-0.28,0.31$, respectively)." should read "carbon concentration and phosphorus concentration ( $\mathrm{r}=-0.31$ and 0.32 , respectively)." 
p. 168 left column, line 3 "0.004" should read " 0.008 ".

p. 169 right column, line 1" 0.47 " should read " 0.49 ".

p. 171 left column, line 5 "- -0.38 " should read " -0.37 ".

Figures with changes:
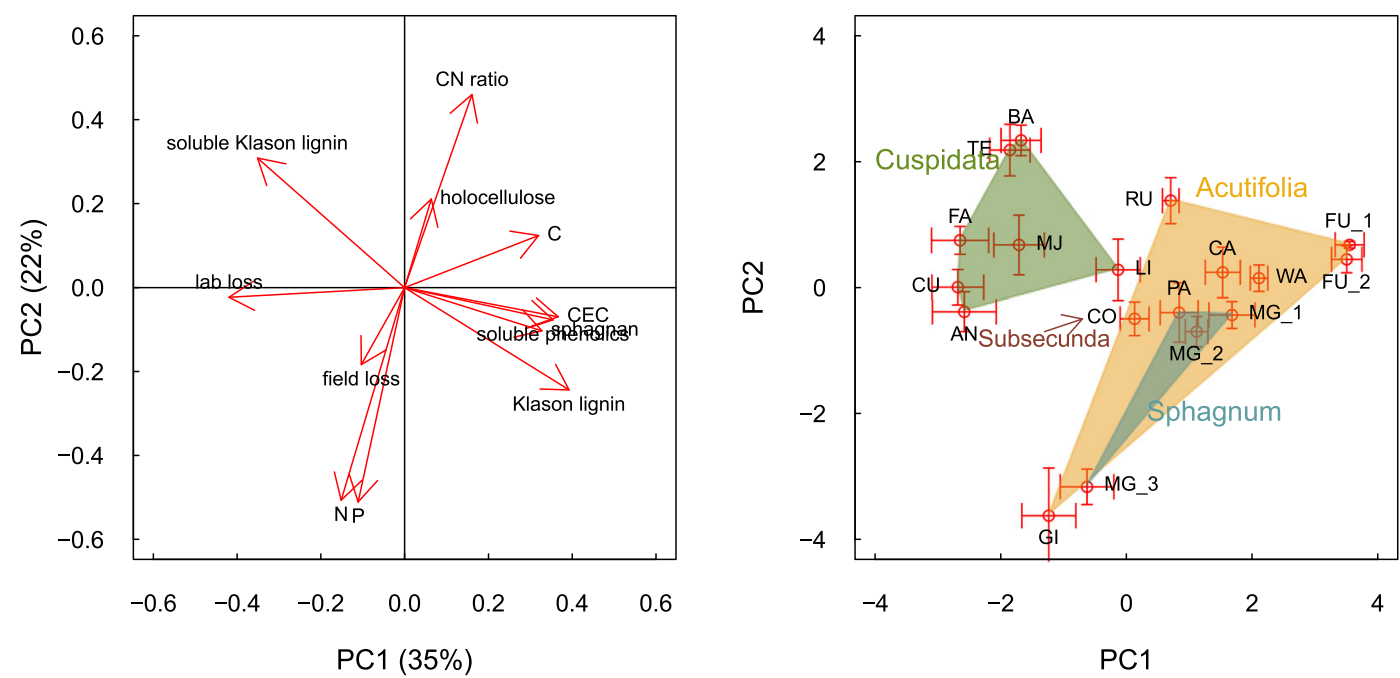

Fig. 3 The variance explained for PC1 is 36\% (not 35\%), for PC2 22\% (not 23\%). The noticeable change in the figure is that FU_1 and FU 2 come closer together

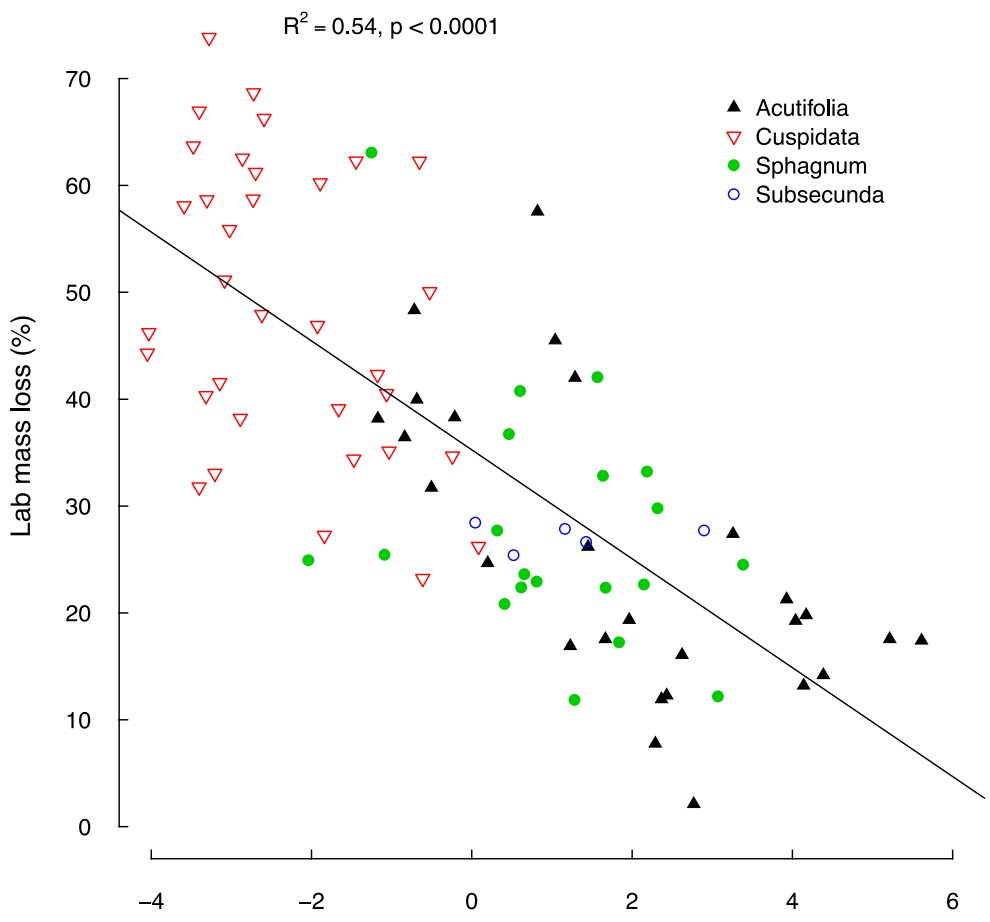

Sum of sphagnan, soluble phenolics, and lignin-like phenolics (scaled)

Fig. 4 Two samples have moved along the $\mathrm{y}$-axis, but $\mathrm{R}^{2}$ - and $p$-values did not change 
Changes in Table 3. The correlations between lab mass loss and other variables were practically the same using the correct data. There were more changes in correlations with $\mathrm{CN}$ ratio. Correlations with $\mathrm{KL}$ and Total KL changed from positive to negative, but remained insignificant; the correlation with soluble phenolics that was reported not significant $(r=0.082)$, became significant $(r=0.23)$; other changes were with $\mathrm{HC}$ (from $r=0.31$ to $r=0.21$ ), CEC (from $r=0.32$ to $r=0.21$ ) and $\mathrm{N}$ (from $\mathrm{r}=-0.94$ to $r=-0.70)$. Other changes were even smaller.

Table 3 Correlations (showing $\mathrm{r}=$ ) among variables describing lab mass loss and concentrations of metabolites and nutrients; bold denotes significant correlation $(P<0.05)$

\begin{tabular}{|c|c|c|c|c|c|c|c|c|c|c|c|}
\hline & $\begin{array}{l}\text { Sphagnan } \\
\mathrm{mg} \mathrm{g}^{-1}\end{array}$ & $\begin{array}{l}\text { Soluble } \\
\text { phenolics } \\
\mathrm{mg} \mathrm{g}^{-1}\end{array}$ & $\begin{array}{l}\text { Klason } \\
\text { lignin } \\
\mathrm{mg} \mathrm{g}^{-1}\end{array}$ & $\begin{array}{l}\text { Soluble } \\
\text { KL } \% \text { of } \\
\text { total KL }\end{array}$ & $\begin{array}{l}\text { Total } \\
\text { Klason } \\
\text { lignin } \\
\mathrm{mg} \mathrm{g}^{-1}\end{array}$ & $\begin{array}{l}\text { CEC } \\
\mu \text { eq } g^{-1}\end{array}$ & $\mathrm{C} \mathrm{mg} \mathrm{g}^{-1}$ & $\mathrm{~N} \mathrm{mg} \mathrm{g}^{-1}$ & $\begin{array}{l}\mathrm{C}: \mathrm{N} \\
\text { ratio }\end{array}$ & $\begin{array}{l}\mathrm{PO}_{4}-\mathrm{P} \\
\mu \mathrm{g} \mathrm{g}^{-1}\end{array}$ & $\begin{array}{l}\text { Lab litter } \\
\text { mass loss } \\
\%\end{array}$ \\
\hline $\begin{array}{l}\text { Holocellulose } \\
\mathrm{mg} \mathrm{g}^{-1}\end{array}$ & 0.268 & -0.071 & -0.027 & 0.113 & -0.004 & 0.268 & -0.103 & -0.330 & 0.210 & -0.160 & -0.074 \\
\hline $\begin{array}{l}\text { Sphagnan } \\
\mathrm{mg} \mathrm{g}^{-1}\end{array}$ & & 0.448 & 0.459 & -0.400 & 0.471 & 0.568 & 0.152 & -0.088 & 0.087 & 0.085 & -0.614 \\
\hline $\begin{array}{l}\text { Soluble } \\
\text { phenolics } \\
\mathrm{mg} \mathrm{g}^{-1}\end{array}$ & & & 0.518 & -0.489 & 0.524 & 0.457 & 0.490 & -0.050 & 0.230 & -0.048 & -0.568 \\
\hline $\begin{array}{l}\text { Klason lignin } \\
\mathrm{mg} \mathrm{g}^{-1}\end{array}$ & & & & -0.940 & 0.998 & 0.573 & 0.468 & 0.012 & -0.038 & 0.046 & -0.588 \\
\hline $\begin{array}{r}\text { Soluble KL\% } \\
\text { of total KL }\end{array}$ & & & & & -0.927 & -0.504 & -0.389 & -0.108 & 0.133 & -0.140 & 0.488 \\
\hline $\begin{array}{l}\text { Total KL } \\
\qquad \mathrm{mg} \mathrm{g}^{-1}\end{array}$ & & & & & & 0.586 & 0.480 & -0.003 & -0.019 & 0.026 & -0.601 \\
\hline CEC $\mu$ eq $g^{-1}$ & & & & & & & 0.279 & -0.229 & 0.213 & 0.093 & -0.619 \\
\hline $\mathrm{C} \mathrm{mg} \mathrm{g}^{-1}$ & & & & & & & & -0.233 & 0.263 & -0.429 & -0.539 \\
\hline $\mathrm{N} \mathrm{mg} \mathrm{g}^{-1}$ & & & & & & & & & -0.698 & 0.699 & 0.237 \\
\hline $\mathrm{C}: \mathrm{N}$ ratio & & & & & & & & & & -0.546 & -0.280 \\
\hline $\mathrm{PO}_{4}-\mathrm{P} \mu \mathrm{g} \mathrm{g}^{-1}$ & & & & & & & & & & & 0.223 \\
\hline
\end{tabular}


Corrected values for C:N ratio and Lab litter mass loss in Supplementary Table 1.

\begin{tabular}{lll}
\hline Species & C:N ratio & Lab litter mass loss $\%$ \\
\hline S. angustifolium & $70.8 \pm 4$ & $63.2 \pm 1.6$ \\
S. balticum & $99.3 \pm 1.5$ & $44.4 \pm 3.9$ \\
S. capillifolium & $88.8 \pm 5.2$ & $17.2 \pm 3.7$ \\
S. contortum & $64 \pm 4.9$ & $27.2 \pm 0.5$ \\
S. cuspidatum & $63.6 \pm 7.6$ & $63.1 \pm 1.6$ \\
S. fallax & $80.7 \pm 3.5$ & $47.1 \pm 3.3$ \\
S. fuscum - bog & $87.2 \pm 4.5$ & $9.0 \pm 2.1$ \\
S. fuscum - fen & $115 \pm 4.6$ & $17.4 \pm 1.2$ \\
S. girgensohnii & $49 \pm 4.9$ & $45.9 \pm 3.5$ \\
S. lindbergii & $71.1 \pm 6$ & $29.1 \pm 2.3$ \\
S. magellanicum -open bog & $76.2 \pm 4.6$ & $24.2 \pm 2.5$ \\
S. magellanicum -pine bog & $68.3 \pm 6.4$ & $18.0 \pm 2.5$ \\
S. magellanicum -spruce forest & $48.3 \pm 2.4$ & $39.4 \pm 6.6$ \\
S. majus & $74 \pm 7.5$ & $48.1 \pm 5.2$ \\
S. papillosum & $73.8 \pm 6.7$ & $29.8 \pm 3.3$ \\
S. rubellum & $97.1 \pm 4.8$ & $38.7 \pm 2.2$ \\
S. tenellum & $89 \pm 4.8$ & $45.3 \pm 7.4$ \\
S. warnstorfii & $74.9 \pm 1.8$ & $20.3 \pm 2$ \\
\hline
\end{tabular}

Corrected models and $\mathrm{R}^{2}$-values in Supplementary Table 3. Models predicting decomposition of Sphagnum litter (mass loss (\%) after 14 months incubation in lab).

\begin{tabular}{|c|c|c|}
\hline Predictors* & Model (intercept+predictor coefficients) & $\mathrm{R}^{2}$ \\
\hline sphagnan & $80.2-1.13 x$ & $\mathrm{R}^{2}=0.38$ \\
\hline soluble phenolics & $66.4-3.88 x$ & $\mathrm{R}^{2}=0.32$ \\
\hline total Klason lignin & $76.5-0.14 \mathrm{x}$ & $\mathrm{R}^{2}=0.36$ \\
\hline sphagnan+soluble phenolics+total KL & $95.9-0.65 \mathrm{x}_{1}-1.74 \mathrm{x}_{2}-0.071 \mathrm{x}_{3}$ & $\mathrm{R}_{\mathrm{adj}}^{2}=0.53$ \\
\hline sphagnan+soluble phenolics & $88.2-0.82 \mathrm{x}_{1}-2.49 \mathrm{x}_{2}$ & $\mathrm{R}_{\text {adj }}^{2}=0.48$ \\
\hline sphagnan+total KL & $93.6-0.78 x_{1}-0.10 x_{2}$ & $\mathrm{R}_{\text {adj }}^{2}=0.49$ \\
\hline sphagnan+soluble phenolics+total $\mathrm{KL}+\mathrm{N}$ & $86.0-0.62 \mathrm{x}_{1}-1.69 \mathrm{x}_{2}-0.074 \mathrm{x}_{3}+1.47 \mathrm{x}_{4}$ & $\mathrm{R}_{\text {adj }}^{2}=0.55$ \\
\hline sphagnan+soluble phenolics+total $\mathrm{KL}+\mathrm{P}$ & $92.1-0.71 \mathrm{x}_{1}-1.55 \mathrm{x}_{2}-0.073 \mathrm{x}_{3}+15.3 \mathrm{x}_{4}$ & $\mathrm{R}_{\mathrm{adj}}^{2}=0.58$ \\
\hline CEC & $94.4-0.083 x$ & $\mathrm{R}^{2}=0.38$ \\
\hline $\mathrm{CEC}+$ soluble phenolics+total $\mathrm{KL}$ & $101.5-0.046 \mathrm{x}_{1}-1.92 \mathrm{x}_{2}-0.062 \mathrm{x}_{3}$ & $\mathrm{R}_{\text {adj }}^{2}=0.51$ \\
\hline CEC + soluble phenolics & $99.0-0.061 \mathrm{x}_{1}-2.48 \mathrm{x}_{2}$ & $\mathrm{R}_{\text {adj }}^{2}=0.48$ \\
\hline $\mathrm{CEC}+$ total $\mathrm{KL}$ & $99.4-0.055 \mathrm{x}_{1}-0.088 \mathrm{x}_{2}$ & $\mathrm{R}_{\text {adj }}^{2}=0.46$ \\
\hline $\mathrm{CEC}+$ soluble phenolics+total $\mathrm{KL}+\mathrm{N}$ & $90.1-0.039 \mathrm{x}_{1}-1.92 \mathrm{x}_{2}-0.069 \mathrm{x}_{3}+1.36 \mathrm{x}_{4}$ & $\mathrm{R}_{\text {adj }}^{2}=0.53$ \\
\hline $\mathrm{CEC}+$ soluble phenolics+total $\mathrm{KL}+\mathrm{P}$ & $97.9-0.051 x_{1}-1.72 x_{2}-0.062 x_{3}+16.25 x_{4}$ & $\mathrm{R}_{\text {adj }}^{2}=0.57$ \\
\hline $\begin{array}{l}\text { Sum of sphagnan, soluble phenolics and total KL (each scaled and centered and } \\
\text { used as one predictor) }\end{array}$ & $35.3-5.1 \mathrm{x}$ & $\mathrm{R}^{2}=0.54$ \\
\hline
\end{tabular}

*Predictor units: CEC in $\mu \mathrm{eq} \mathrm{g}^{-1}$, all other predictors in $\mathrm{mg} \mathrm{g}^{-1}$

Publisher's note Springer Nature remains neutral with regard to jurisdictional claims in published maps and institutional affiliations. 